\title{
Telenursing in colorectal cancer patient follow-up and treatment assessment: a mixed methods evaluation study
}

\author{
Maria José Dias, Maria Fragoso, Lúcio Lara-Santos \\ Instituto Português de Oncologia, Francisco Gentil, Porto \\ \{maria.mjsdias,llarasantos@\}gmail.com, \\ carocha321@hotmail.com
}

\author{
Pedro Pereira Rodrigues \\ LIAAD - INESC TEC \& \\ CINTESIS, Faculty of Medicine, \\ University of Porto
}

pprodrigues@med.up.pt

\begin{abstract}
The incidence of colorectal cancer cases in the Portuguese Institute of Oncology of Porto created the need of a telenursing program in the Gastro-Intestinal Cancer Unit. After staging, treatment may involve surgery radio and chemotherapy (either oral or IV). Patients with no treatment after surgery are scheduled for medical exams every 3 months in the first 2 years. Patients on chemotherapy need to be compliant and to have a close monitoring of adverse events. The GI Cancer Unit uses a telenursing information system to help assess colorectal cancer patients' follow-up after surgery, medical treatment compliance and adverse events. A mixed-methods evaluation was done to a) describe the target population, b) detect problems in the telenursing information system, and c) suggest changes to meet users' requirements. From 181 outbound phone calls, representing 67 patients (49 in treatment and 18 in follow-up), patients' main characteristics were extracted and system's problems were identified by the intervening nurses. Recommendations will be useful for a further development of the system.
\end{abstract}

\section{Introduction}

In 2009 the most frequent cause of admission to the Portuguese Institute of Oncology of Porto was digestive cancer $(23.4 \%$ of admissions) [1]. Of all admitted patients in 2007, 6.4\% had colon cancer and $5.9 \%$ had rectum (total of 2197 colorectal cancer CRC patients) [2].

Staging, meaning evaluation of the clinical extension of cancer, follows clinical diagnosis.

The TNM Classification of Malignant Tumours (TNM) describes the extent of a person's cancer, and uses $\mathrm{T}$ to describe the size of the original (primary) tumor and whether it has invaded nearby tissue $\mathrm{N}$ to describe nearby (regional) lymph nodes that are involved, $\mathrm{M}$ to describe distant metastasis (spread of cancer from one part of the body to another) [3].

Treatment is decided after clinical staging. Treatment options may include surgery, radio and chemotherapy under different sequences and combinations. Surgery may be the one only option for patients who are surgery pathological specimens N0, as they have no lymph nodes involved by the cancer, or who have comorbidities that do not allow chemotherapy and radiotherapy regimens to be prescribed. These patients are traditionally followed in our clinical unit out-hospital ward every 3 months, after end of treatment, according to international follow-up guidelines.During these medical follow-up evaluations, a clinical exam is performed and blood samples are collected for hematology, biochemistry and CEA-Carcinoembryonic Antigen).

After starting our telenursing program, surgical only patients, (group A), were no longer required to come to Hospital at 3 months after surgery, for medical examination, unless they had any complaints and /or CEA increase.

For these patients phone monitoring allows:

- general complaints;

- digestive complaints;

- medication;

- comorbidities;

- telling the patient that he /she has a normal/abnormal CEA; in the latter the patient is instructed to get to the hospital for medical observation within a week;

- family and patient education,

- data collection. 
Chemotherapy may be used in quite different settings; using it after curative surgery, aims to increase survival and disease free survival (adjuvant treatment); in rectal cancer we use it with radiotherapy, trying to shrink the tumor, making it easier to resect and thus reduce the need for a definitive ostomy (neoadjuvant treatment). Whenever CRC is disseminated into other organs other than colon /rectum, the disease is treated on a palliative setting (palliative treatment).

Chemotherapy provides better and better results and its use is increasing [4]. However these treatments affect patients' body image, physical and psychological health, sexuality, and therefore quality of life [5] having a profound impact on patients, families and society [6].

Usually, patients have to manage chemotherapy adverse events at home without professional health care support or any other [6]. These events if not properly and early identified, can turn out to be critical for the patient, as may occur with toxic diarrhea, mucositis, fever and neutropenia [7].

A simple, yet effective, solution may be using communication and technology developments and try to improve the quality of care, as we may teach patients to self-manage their disease-related complaints, and treatment adverse events. For these patients, (Group B), phone monitoring allows:

- adverse events and symptom monitoring,

- data collection,

- interventions to be carried out at home,

- family and patient education

- Multidisciplinary monitoring for these interventions [8].

\subsection{Health information systems in cancer treatment}

Some health information systems are already in use, in hospitals, for cancer treatment adverse events monitoring. Examples of such health information system include ASyMSB (advanced symptom management system) [19] and ISSAC (interactive symptom assessment and collection). ASyMSB is used in English hospitals, for adverse events management [10]. The patient daily reports the adverse events answering a survey by using a PDA $[11,12]$. These events are then evaluated by the memorial Symptom Assessment Scale [8]. ISSAC is a web tool used in hospitals but it can also be accessed by patient through the internet. The survey has a 0 to 10 , scale and when the survey score is higher than a threshold, health professionals are notified by email and proceed with calling the patient.

\subsection{Evaluation using ethnographic and qualitative studies}

Usually, strict study designs are required to properly evaluate interventions. However, some evaluations require more pragmatic and flexible approaches for the production of evidence, than those allowed within the rigid structures of controlled study designs. Methodological issues can thus come from that need, in defining and measuring clinical practice, and from the difficulty in interpreting study findings [13].

Ethnographic studies use various qualitative research techniques to obtain data from several sources and integrate them in actual findings. The main idea behind ethnographic studies is that the researcher, during an extended period of time, collects informal data to promote the effective evaluation of the intervention, in particular, telehealthcare [14]. This data gathering is based mainly on informal observations and discussion with users for problem detection, and qualitative question answering to recommend improvements.

\subsection{The telenursing program}

Telenursing is the use of "technology to deliver nursing care and conduct nursing practice" [15]. Our telenursing program uses phone calls, in a selected group of CRC patients. Nurses engaged in telenursing practice continue to assess, plan, intervene, and evaluate the outcomes of nursing care.

The target population definition included 2 different groups:

Group A included patients submitted to surgery, with no lymph nodes involved by cancer disease in the pathological specimen or with comorbidities not allowing chemotherapy. These patients will attend a medical consultation within 6 months provided they have neither complaints nor CEA increase at 3 months after surgery; if only one of these occurs, medical advice will be provided.

This group cases are selected during multidisciplinary decision meeting; if they accept to be part of the program they will be called by a nurse at 3 months, approximately 1 week after taking a blood sample for CEA measurement; the lab result will be provided and if within normal limits the patient will have to come to hospital at 6 months as previously scheduled. If not, or if the patient has complaints related to the surgical procedure, he will be seen by a doctor shortly after the call.

Group B included all CRC patients that are bound to start on chemotherapy whatever setting or regimen; they are selected at the clinical nurses consultation 
after doctor's examination and prescription of chemo. They will get chemotherapy instructions and signed informed consent for the program will be requested at this moment.

Phone call schedule is different according to chemotherapy treatment.

- Systemic (intravenous) treatment: 1 week after treatment start, and then as needed according to patient's complaints

- Oral treatment: one call up to day three, and then weekly; calls may be done on as needed basis if the complaints justifies it

- Neoadjuvant (before surgery): weekly after cytotoxic treatment starts.

During chemo, patients attend the hospital, usually every three weeks. During chemo radiotherapy, they are closely followed every week.

After calling the patient, the nurse must register all relevant information; symptoms, compliance for oral chemo and whatever questions might have been asked.

Both groups are informed about procedures and what they are expected to inform [16].

Both calls are registered in the unit database.

Four nurses ran the program; they went through three months training, including topics on cancer patient's psychology, effective communication, informatics (related to the information system in use), and guidelines structuring.

\section{Telenursing support system}

The development of this support system had an endpoint - the psychological well-being of cancer patients (Figure 1).

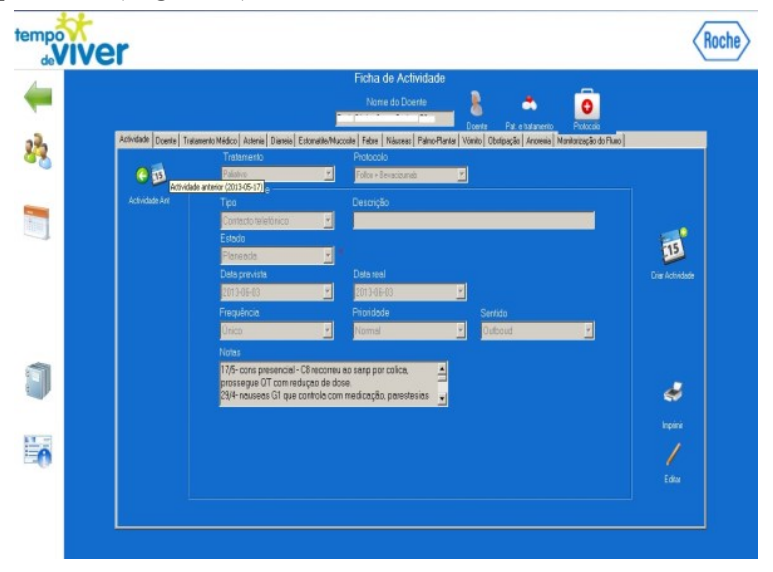

Figure 1. Telenursing support system

After testing the first draft the information system got improved, including the upgrade of data collection, improvement of information accessibility and inclusion of structured guidelines, to enable a more comprehensive and sustainable support to patients. The inclusion of guidelines to help the nurse activity was of utmost importance (Figure 2). The system will include algorithms for surgery follow up and for chemotherapy and radiotherapy adverse events.

For group A the program intends to help reduce the number of patient's visits to the Hospital, without increasing the risk of undetected recurrence.

For group B patients our intention was reducing the number of sustained serious toxicities secondary to the treatment, as the patient often does not come to the hospital in due time.

Currently, only the guideline dealing with surgery follow up and chemotherapy treatment are in use.

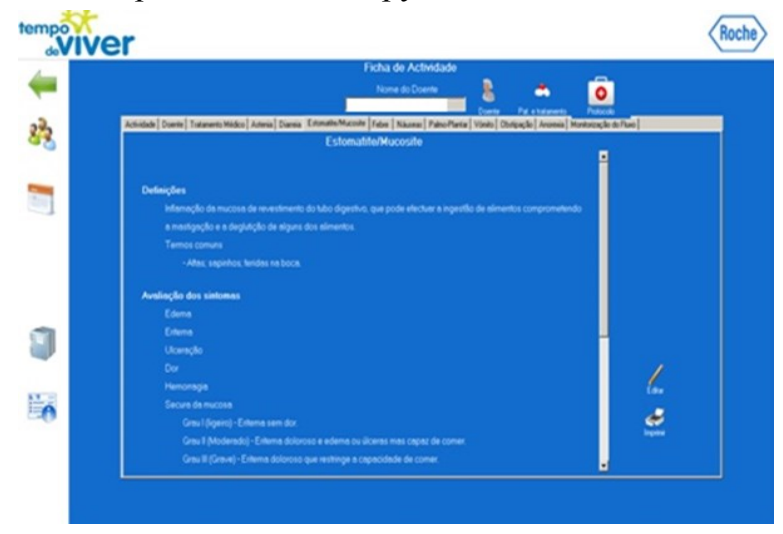

Figure 2 - support system guideline

\section{Aim}

This work aimed as performing a mixed-methods study to evaluate the telenursing information system. Currently it is being used to help assessing colo-rectal cancer patients in treatment, monitoring adverse effects of chemotherapy treatment and follow up patients. The telenursing program was accomplished in a oncologicdedicated hospital.

Evaluation was conduced to a) describe the target population, b) detect problems of the telenursing information system and c) suggest changes to meet the user requirements.

This work's endpoint was increasing quality of care. The quality audit was made through the creation of a data base and analysis of follow-up data for both groups of patients. Then telenursing information system was evaluated.

Follow-up data were retrieved from clinical files, and were defined as relapse occurrence for group A patients and toxicity data for group B.

\section{Material and Methods}

\subsection{Population}


Colorectal Cancer patients, followed in the GI Cancer Unit are evaluated at the nurse's consult at surgery follow up or before chemotherapy treatment; signed informed consent is requested for them to be included in the study. Provision of personal or care provider mobile and home phones are registered.

\subsection{Sampling strategy}

The sample was a consecutive one, including every patient within a 3 month period gathered by convenience (March to May 2011).

\subsection{Variables/data strategy}

Registered data for Group A included symptoms and questions reported by the patient/caregiver, and CEA laboratory value; for Group B chemotherapy treatment protocols, cancer stage, adverse events were collected and registered.

In the mixed-methods study the data were collected by informal interviews, by phone, and direct information from system users; interaction was made with the nurses involved in the program.

\subsection{Data analysis}

Absolute and relative frequencies were used to describe categorical variables, while median and percentiles were used for continuous variables. Pearson's chi-squared test was used to compare proportions of different groups.

\section{Results}

A total of 181 outbound phone calls were included, representing 67 patients (49 in treatment and 18 in follow-up).

There is one phone call to a stage 0 case, due to the fact that he had a neuropsychiatric condition and needed professional support.

There is also one patient that was later excluded from the study as he was on combination chemotherapy, both oral and IV.

\subsection{Sample description}

Group A included 18 patients; $81.3 \%$ had a normal value of CEA at 3 months, no complaints, and kept their scheduled visit 3 months later. Three patients had an earlier visit scheduled due to increased CEA, clinical complaints or both.

The median age was 64 for both groups (P25:54, P75:69), 75 for group A and 61 for group B. More than half $(69 \%)$ of the patients were male. Only $27 \%$ of them were in follow up after surgery. (Table I)

As for tumor topography, $58 \%$ of the patients had colon cancer while $42 \%$ had rectum cancer $(\mathrm{p}=0.774)$. The prevalence/stage was stage II $39 \%$, stage III $27 \%$ and stage IV 36\%. (Table II)

There are patients on stage II, that went to chemotherapy; there were 24 stage II patients and only 18 Group A Cases.

Out of the $49(73 \%)$ patients on chemotherapy, $57 \%$ were in palliative, $22 \%$ were in neoadjuvant, and $21 \%$ in adjuvant treatment. (Table III)

Irrespective of the protocol setting $32 \%$ of the patients, were in oral chemotherapy treatment, with capecitabine.

Out of the 181 calls, 132 were performed to patients on chemotherapy treatment, $24 \%$ of which to patients on oral treatment. (16 patients)

Most calls were protocol related and for capecitabine treatment compliance was questioned; supportive medication as antiemetic, analgesics and oral mouthwash were not taken as prescribed in $87 \%$ of the cases, $\mathrm{p}<0.001)$, Patients who reported adverse events had more extra calls ( $89 \%$ vs. $62 \%, \mathrm{p}=0.021)$.

For patients having the extra call unexpected adverse events were registered in more than half $(66 \%$, $\mathrm{p}<0.001)$ of the calls; they were more frequent with systemic treatment than oral treatment (48\% vs. $71 \%$, $\mathrm{p}=0.046)$;

Treatment toxicities events come at different times, depending on the treatment protocol. Therefore only the first call for oral or for systemic treatment was always evaluated.

Most call registries $(75 \%, \quad \mathrm{p}<0.001)$ had information regarding side events (present or not present); missing data related to the seriousness of the event were lower for systemic treatment (69\% vs. 94\%, $\mathrm{p}=0.006$ ).

\subsection{Results from the mixed-methods study}

During the three months of the data collection we kept a debate with the users in order to detect difficulties. They were divided into major and minor difficulties.

\subsubsection{Major difficulties}

Major difficulties had to do with:

- The hospital information system and the telenursing support system are not able to share information. For example, it is not possible, during a phone call, to access information regarding either administrative or clinical data. 
- If a change of protocol occurs information from previous registries will be lost (clinical data and performed calls).

- There is no specific data entry form to register systemic chemotherapy adverse events.

- There are no guidelines specific for each treatment protocol.

- Although not researched specifically, patient safety is an important part of the diagnosis, monitoring, outcomes, and technical tools used in telehealth practice.

\subsubsection{Minor difficulties}

Minor Difficulties had to do with:

- creating a new registry for a new patient;

- registering chemotherapy protocol;

- registering every phone call, either planned or unplanned;

- not having guidelines for the operative system

- not being able to register significant and common pathologies;

- relevant information such as the name of an important care provider cannot be registered;

- user can introduce information regarding contact time preference (day or night) but the system, allows contact at any time of the day. Nonclinically relevant information such as address and zip code are considered as mandatory;

- clinically relevant information, such as first day of treatment and date of diagnosis is not mandatory;

- first treatment date is most of the times not completed by users because it takes them too long to get it from the hospital system.

\section{Discussion}

The sample is a small one, but similar to the published literature, as far as group B is analyzed, regarding epidemiology and adverse events of cancer treatment $[3,5]$. We found no articles to describe the nurse's 3 months consultation with CEA for patients in follow up (group A).

The Palliative setting of treatment is increasing, as we have many more treatment options than we used to; new treatments lead to better survivals, and thus increasing number of patients in this setting (36\% of patients have metastatic disease as they are selected). Neoadjuvant treatment is prescribed for locally advancer rectal cancer that represents almost half of the cases, as expected from the North Cancer Registry.
A possible explanation for lower median age for group B patients is the fact that risk associated comorbidities increase with patients'age.

There were patients with no lymph nodes involved that went on to chemotherapy; this fact is explained by the existence of a high-risk group among stage II patients that have benefit when submitted to adjuvant treatment.

Oral chemotherapy is used increasingly, as it demonstrated non inferiority when compared to intravenous drugs, while improving quality of life and toxicity profile.

Chemotherapy adverse events are very common although not always clinically significant; however, as less toxicity through treatment was an endpoint, extra calls were made, on as needed basis, to patients reporting more serious adverse events.

Higher quality of data collection for oral treatment may be explained by the fact that the whole system has been put in place for this particular treatment.

When we looked back at the system, several recommendations could be produced:

- Medical visit and treatment dates should be immediately available for every patient. A calendar overview of all the schedules visits would allow a computer assisted phone call.

- Gathered information should not be lost under any circumstances, and, as cancer is a dynamic disease, the program must be user friendly and allow protocol specifications changes when the patient moves from one therapeutic setting to another.

- Algorithms per protocol must be published, and regular revisions made. As there are important differences among different treatments and as all collected data are being entered into a field created for oral chemotherapy adverse events, reliability on the data produced is decreased.

- A scoring system, such as CTCAE (Common terminology cancer adverse events) might be of use for similar registries by different nurses

- To overcome the interface problems, the conversation flow should resemble the nurse patient interview.

- Registration fields should appear only if necessary in each part of the conversation flow. If the patient reports a specific adverse effect (e.g. mucositis) the user does not need other problems' guidelines (e.g. diarrhea).

- Guidelines should become visible to the user only according to protocol.

- Users have straight access to patients' demographic information through hospital system; thus demographic data should not be 
mandatory. This item is time and human resource consuming and not needed as our main objective is side event control.

- The system should provide a guideline to the user at the time the patient is included, per protocol and activity.

For minor problems we may suggest updating common pathologies, and care provider identification; information completeness (mandatory versus optional) should also be reappraised.

The safety issues associated with care delivered using telecommunications technologies have not been an issue, but future work should consider it.

\section{Main findings and recommendation}

This application is considered innovative in cancer population and, to our knowledge, we were the first center to use it. The telenursing system includes more than one area of activity.

Patients with low risk of relapsing disease after surgery are probably spared unneeded hospital visits; hospital and family costs are reduced, and it seems the patient is not jeopardized.

Chemotherapy treatment adverse events may be supported by nurses' advice at an early phase. However patients are instructed to come to hospital if the event persists or gets worse. This way safety remains within limits.

In the future we might monitor adverse events of radiotherapy treatment and make the follow-up of fasttrack surgery [16], as guidelines are already prepared. The study suggests subjective benefit for most of the patients and better communication between health professionals and patients as described in literature [12].

Significant failures were detected in the system; tools' alteration would assist the work related to the phone calls activity. For the future we are working on the system to improve data collection and registry. The information system can be extended being able to support guidelines and data collection for other cancer units.

In general, the patients' feedback was positive (not quantified).Patients' quantitative evaluation should be included

Although not noted or researched specifically, patient safety is an important part of the diagnosis, monitoring, outcomes, and technical tools used in telehealth practice. Future investigation should monitor safety.

As a summary we would say that technologies have evolved to offer more and broader capability for telehealth/telenursing practice.

\section{Acknowledgments}

Emília Alves, Paula Mendes, Armanda Nogueira, Susana Santos and Fátima Teixeira are acknowledged for their participation in the Project.

\section{References}

[1] Melo M, Braga R. (2003). Rastreio do Cancro do Cólon e do Recto. Rev Port Clin Geral 19:471-82.

[2] Pontes L, Bento MJ. Registo Oncológico. (2007), Instituto Português de Oncologia de Francisco Gentil, Centro Regional de Oncologia do Porto, EPE, 2009.

[3] Ajcc - American joit committee on cancer (2010). Cancer staging handbook: International union against cancer. 52-80

[4] Scottish Executive (2001) Cancer scenarios: an aid to planning cancer services in Scotland in the next decade. Access at 20 august 2011.

[5] C-Couveur. (2001). A qualidade de vida: Arte para Viver no Século XXI. Loures. Lusociência.

http://www.scotland.gov.uk/Resource/Doc/158659/0043045. pdf.

[6] Grady J, Schlachta-Fairchild L, Elfrink V., (2004). The 2004 International Telenursing Role Survey. Abstracts from The American Telemedicine Association Tenth Annual Meeting \& Exposition April Telemed e-Health $J, 11(2): 197$.

[7] Department of Health. (2006), Our Health, Our Care, Our Say: a New Direction for Community Services.

[8] Kearney N, Kidd L, Miller M, et al., (2006). Utilising handheld computers to monitor and support patients receiving chemotherapy: results of a UK-based feasibility study. Support Care Cancer: 14(7):742Y752.

[9] McCann- Maguire R- Miller M, et al., (2009). N. Patients' perceptions and experiences of using a mobile phone-based advanced symptom management system (ASyMSB) to monitor a manage chemotherapy related toxicity. Eur J Cancer Care; 18:156Y164

[10]Cancer care Ontario: University Avenue Toronto Ontario, Canada; Access 20 October 2010: http://www.cancercare.on.ca/ .

[11] Kearney N, McCann L, Norrie J, et al., (2009) Evaluation of a mobile phone based, advanced symptom management system (ASyMSB) in the management of chemotherapy related toxicity. Support Care Cancer: 17-43.

[12] R-Maguire, M-Miller, Sage. (2005). Results of a UK based pilot study of a mobile phone based advanced symptom management system (ASyMS) in the remote monitoring of chemotherapy related toxicity. Clinical Effectiveness in Nursing 9 (3-4), 202-210.

[13] Hammersley M, Atkinson P. Ethnography: principles in practice. London: Routledge, 1995

[14] Finch T, May,C. Integrating service with evaluation in telehealthcare: an ethnographic study: BMJ 2003:327

[15] Fairchild, L, Elfrink. (2008) Patient safety, telenursing, and telehealth: An Evidence-Based Handbook for Nurses: Vol. 3

[16] Wind J, Hofland, et al. (2006) Perioperative stategy in colonic surgery; laparoscopy and /or fast track multimodal management versus standard care (LAFA trial), BMC Surg. 2006. 